\title{
Ley № 20.005 sobre Acoso Sexual en Chile
}

Patsilí Toledo*

\section{Antecedentes}

A partir de la reapertura del Congreso Nacional en 1990, como consecuencia del restablecimiento de la democracia, fueron presentados numerosos proyectos de ley relativos a la situación de las mujeres en Chile ${ }^{1}$. Varios de ellos tendían a reconocer en el ordenamiento jurídico nacional formas específicas de violaciones a sus derechos humanos y a adecuar la legislación -que había experimentado incluso retrocesos en esta materia durante la dictadura- a los avances ya consagrados en el derecho comparado y a los nuevos estándares de derechos humanos.

En 1994, con un apoyo parlamentario transversal aunque no amplio, se inició la tramitación del Proyecto de Ley sobre Acoso Sexual que llegaría a convertirse en ley en el año 2005. La Ley № 20.005 -“Tipifica y sanciona el acoso sexual" - fue promulgada el 8 de marzo de 2005, luego de más de diez años de tramitación parlamentaria ${ }^{2}$, modificando e incorporando diversas disposiciones al Código del Trabajo, el Estatuto Administrativo y el Estatuto Administrativo para Funcionarios Municipales. Fue publicada en el Diario Oficial el 18 de marzo del mismo año.

\section{Acoso sexual y Derecho Internacional de los Derechos Humanos}

El hostigamiento o acoso sexual ha sido abordado en el ámbito de los derechos humanos como una de las manifestaciones de la violencia contra las mujeres que vulnera múltiples derechos fundamentales. Si bien en términos teóricos es posible sostener que la violencia contra las mujeres siempre ha constituido un atentado a los derechos humanos de éstas, sólo a partir de la década pasada esta realidad comenzó a ser abordada en el ámbito del Derecho Internacional de los Derechos Humanos, incorporándose en declaraciones, convenciones y dentro de los mecanismos de protección de los sistemas universal e interamericano de derechos humanos ${ }^{3}$.

En efecto, el trabajo en derechos humanos de las mujeres ha requerido de grandes esfuerzos para lograr el reconocimiento de los atentados a estos como verdaderas violaciones de derechos humanos. Las postergaciones y dificultades para el avance en esta temática han sido justificadas sobre la base del carácter "cultural" de estas violaciones que, en consecuencia, sólo serían superadas con la evolución de las sociedades y el cambio cultural. Así, aunque los derechos a la igualdad y no discriminación, a la integridad física y psíquica de las personas, entre otros, han sido reconocidos universalmente, tratándose del respeto y

* Abogada, Corporación Humanas-Centro Regional de Derechos Humanos y Justicia de Género. Profesora del curso "Género y Derecho" en la Facultad de Derecho, Universidad de Chile.

1 En 1991 fue presentado el proyecto de ley que daría lugar, en 1994, a la llamada Ley de Violencia Intrafamiliar (№ 19.325); en 1992 se presenta el primer proyecto que "Establece derecho a indemnización por acoso sexual" (Boletín 583-07). Además, en mayo de 1990 se presentó el proyecto de ley que creó el Servicio Nacional de la Mujer (Ley № 19.023).

2 Boletín № 1419-07, de 24 de octubre de 1994.

3 Por ejemplo, Declaración y Programa de Acción de Viena (1993); Declaración sobre la Eliminación de la Violencia contra la Mujer (1993); Convención Interamericana para Prevenir, Sancionar y Erradicar la Violencia contra la Mujer (1994);

Este comentario, junto con el documento principal a que se refiere, están disponibles en www.anuariocdh.uchile.cl 
creación de la Relatoría Especial sobre la Violencia contra la Mujer (1994); y otras.

4 Diario Oficial, 11 de Noviembre de 1998.

5 Naciones Unidas, Consejo Económico y Social. Informe preliminar presentado por la Relatora Especial sobre la violencia contra la mujer, con inclusión de sus causas y consecuencias, Sra. Radhika

Coomaraswamy, de conformidad con la resolución 1994/45 de la Comisión de Derechos Humanos, párr. 190.

6 Observación General Nº 19 del Comité para la Eliminación de la Discriminación contra la Mujer, 1992.

7 Diario Oficial, 9 de diciembre de 1989

8 Este artículo señala “...toda distinción, exclusión o restricción basada en el sexo que tenga por objeto o por resultado menoscabar o anular el reconocimiento, goce o ejercicio por la mujer, independientemente de su estado civil, sobre la base de la igualdad del hombre y la mujer, de los derechos humanos y las libertades fundamentales en las esferas política, económica, social, cultural y civil o en cualquier otra esfera". garantía de estos derechos a las mujeres, se ha requerido de una mayor tenacidad por parte de quienes los promueven; pero los progresos han sido limitados y recientes.

El acoso u hostigamiento sexual es, aún más claramente, un fenómeno reciente en términos históricos, asociado a la reproducción de la discriminación de las mujeres en los nuevos espacios en que éstas comienzan a desenvolverse, tales como el trabajo, la educación y la política. Sin embargo, desde una perspectiva más amplia, podemos decir que el hostigamiento sexual siempre ha constituido una forma de excluir veladamente a las mujeres de los espacios públicos, haciendo primar su rol sexual y reforzando su adscripción al espacio doméstico, en contraposición a un espacio público amenazante. De allí se desprende que una de las formas más antiguas y generalizadas de hostigamiento sexual contra las mujeres es el que se produce en las calles, espacio público por antonomasia.

De acuerdo a la Convención Interamericana para Prevenir, Sancionar y Erradicar la Violencia contra la Mujer (Convención de Belém do Pará) ${ }^{4}$, "debe entenderse por violencia contra la mujer cualquier acción o conducta, basada en su género, que cause muerte, daño o sufrimiento físico, sexual o psicológico a la mujer, tanto en el ámbito público como en el privado". De acuerdo a la misma convención, el acoso sexual es una de las formas de violencia contra la mujer que tiene lugar en la comunidad, incluyendo el lugar de trabajo, las instituciones educativas, establecimientos de salud y cualquier otro lugar.

El acoso sexual -entendido como una conducta o atención sexual no deseada por la persona que la recibe y que resulta ofensiva o amenazadora para ésta ${ }^{5}$ - así como otras formas de violencia contra las mujeres, no sólo constituyen atentados contra su integridad física y psíquica, sino también son las más severas manifestaciones de la discriminación contra ellas. El Comité para la Eliminación de la Discriminación contra la Mujer ha señalado que la violencia contra las mujeres, en la medida que las afecta por el solo hecho de ser tales o que las afecta en forma desproporcionada, constituye una forma de discriminación ${ }^{6}$ en los términos del artículo 1 de la Convención sobre la Eliminación de Todas las Formas de Discriminación contra la Mujer ${ }^{7}$ (CEDAW, por sus siglas en inglés).

Efectivamente, si tomamos como base la definición de discriminación contra la mujer establecida en el artículo 1 de la CEDAW ${ }^{8}$, la violencia y, en particular, el acoso sexual, constituyen formas de discriminación contra las mujeres, en cuanto importan una distinción de trato basada en el sexo, que tiene por resultado menoscabar el goce y ejercicio de los derechos humanos y libertades fundamentales de las mujeres.

La multiplicidad de ámbitos en los que se presenta el hostigamiento sexual -laboral, educacional, político y otros- determina también la diversidad de los derechos humanos que resultan menoscabados como consecuencia de esta conducta. En este sentido, y si bien todas las formas de violencia contra las mujeres afectan el goce y ejercicio de sus derechos en todos 
los ámbitos, el caso del hostigamiento sexual tiene efectos especialmente en los derechos económicos, sociales y culturales de las mujeres. El derecho al trabajo y a la educación, por ejemplo, se ven vulnerados no sólo en la medida que estas conductas inducen a las mujeres a abandonar sus empleos o estudios, sino también en cuanto implican un trato discriminatorio en el ejercicio de esos derechos.

Al respecto, la Relatora Especial sobre la Violencia contra la Mujer ha señalado que "[e]l acoso sexual de la mujer en el lugar de trabajo, en los centros docentes y otros lugares, atribuye un papel sexual a la mujer y contribuye a perpetuar su subordinación en la sociedad. El acoso sexual constituye una forma de discriminación sexual, pues no sólo degrada a la mujer, sino que refuerza y refleja el concepto de falta de profesionalismo por parte de las trabajadoras a las que, en consecuencia, se considera menos aptas para realizar sus tareas que sus colegas masculinos ${ }^{\prime \prime}$.

\section{Principales aspectos de la legislación sobre acoso sexual en relación a las obligaciones internacionales del Estado chileno en la materia}

En las Observaciones Finales del Comité de Derechos Humanos respecto del cuarto informe periódico de Chile, en 1999, el Comité manifestó su preocupación por "el elevado número de casos de hostigamiento sexual en el lugar de trabajo", recomendando al Estado "que se promulgue una ley tipificando el delito de hostigamiento sexual en el lugar de trabajo"10. También en 1999, el Comité para la Eliminación de la Discriminación contra la Mujer solicitó al Estado la inclusión, en su próximo informe, de datos relativos a "la condición de las mujeres trabajadoras y el avance en sus condiciones de vida, y acerca de las facilidades para el cuidado de los niños y el problema del acoso sexual en el trabajo" ${ }^{11}$.

La Ley № 20.005, finalmente, no tipificó como delito el hostigamiento sexual en el lugar de trabajo -tal como lo recomendó el Comité de Derechos Humanos en 1999-, aunque el proyecto de ley original sí establecía la sanción penal de esta conducta ${ }^{12}$. Con ello, igualmente, se facilitó la exclusión del debate legislativo de la posibilidad de incorporar otros ámbitos fuera del laboral en los que se puede presentar el acoso sexual ${ }^{13}$.

La norma matriz de la ley se cristaliza en el nuevo inciso segundo del artículo 2 del Código del Trabajo, estableciendo que "las relaciones laborales deberán siempre fundarse en un trato compatible con la dignidad de la persona", señalando al acoso sexual como una de las conductas contrarias a ella. La relevancia de la disposición, evidentemente, es mucho mayor que la sola incorporación del hostigamiento sexual como conducta reprochable en el ámbito laboral. Con ella, se reconoce formalmente el trato acorde con la dignidad de la persona -junto con el reconocimiento de la función social del trabajo, la libertad de contratación y la prohibición de la discriminación- como uno de los principios básicos del Derecho Laboral en nuestra legislación.

La ley define el acoso sexual como "el que una persona realice, en forma indebida, por cualquier medio, requerimientos de carácter sexual, no
9 Naciones Unidas, op. cit. párr. 202.

10 Observaciones finales del Comité de Derechos Humanos: Chile. 30/03/99 CCPR/C/79/Add.104 (Concluding Observations/ Comments), párr. 18.

11 Observaciones finales del Comité para la Eliminación de la Discriminación contra la Mujer: Chile. 09/07/ 99. A/54/38, paras.202-235. (Concluding Observations/ Comments). Párr. 11 (Recomendaciones).

12 Se tipificaba como una falta, incorporada en el artículo 494 del Código Penal. Esta propuesta fue eliminada por la Comisión de Constitución, Legislación y Justicia de la Cámara.

${ }^{13}$ Cabe hacer presente que, desde el proyecto original, siempre se limitó el fenómeno al hostigamiento sexual en el lugar de trabajo, excluyéndose otros ámbitos -como el académico-, a pesar de que diversas parlamentarias hicieron presente este punto durante la tramitación legislativa del proyecto. 
${ }^{14}$ Artículo 2 inciso $2^{\circ}$ del Código del Trabajo.

15 En primer término, excluye del concepto la eventual existencia de una relación jerárquica entre los sujetos activo y pasivo del acoso, mientras que en el proyecto inicial sólo podía ser sujeto activo el empleador o un superior jerárquico. En segundo término, la expresión

"requerimientos de carácter sexual" empleada por la ley abarca más situaciones de hostigamiento sexual que "solici[tar] verbal o gesticularmente relaciones sexuales, contacto sexual o cualquier otra forma de actividad sexual", que eran las conductas que comprendía el proyecto en su versión original. Finalmente, de acuerdo al proyecto de ley originario, debía existir una amenaza o promesa expresa de un trato preferencial o perjudicial en el empleo, asociado a aquellas solicitudes. En este último aspecto, el tenor de la ley permite contener una mayor cantidad de hipótesis comisivas de este ilícito laboral, en especial cuando se presenta entre personas no vinculadas jerárquicamente.

16 Artículo 153 inciso $1^{\circ}$ del Código del Trabajo.

17 Artículo 153 inciso 2o del Código del Trabajo.

18 Artículo 154 № 12 del Código del Trabajo.

19 Título IV del Libro II del Código del Trabajo. consentidos por quien los recibe y que amenacen o perjudiquen su situación laboral o sus oportunidades de empleo"114. El concepto, si bien supera en varios sentidos el contenido en el proyecto de ley original' ${ }^{15}$, igualmente excluye, con la noción "requerimientos", otras hipótesis de acoso sexual que consisten en atenciones sexuales no bienvenidas y que resultan ofensivas o amenazantes para la víctima, aunque no constituyan "requerimientos". Considerando la levedad de las sanciones mínimas que establece la ley -como se señala más adelante- resulta contradictorio que estas acciones menos graves, pero no por eso inocuas, se excluyan del concepto.

Un aspecto que merece mayor atención es el relativo a los procedimientos de investigación, ya que la ley delega en las propias empresas, de manera sustantiva, las funciones de investigación y sanción. En efecto, la nueva ley establece que, tratándose de empresas obligadas a confeccionar reglamentos internos ${ }^{16}$, están obligadas a incorporar en ellos las normas que garanticen un ambiente laboral digno y de mutuo respeto entre los trabajadores ${ }^{17}$, así como el procedimiento, medidas de resguardo y sanciones que se aplicarán en casos de denuncias de acoso sexual ${ }^{18}$.

El procedimiento de carácter general frente a este tipo de denuncias ${ }^{19}$ sólo puede iniciarse a través de la denuncia por escrito de la persona afectada, presentada dentro de la empresa o en la Inspección del Trabajo respectiva, a elección de quien la formula. En este punto cabe destacar que la denuncia sólo puede ser realizada por la persona directamente afectada, lo cual no se condice con la obligación de garantizar un ambiente laboral digno y de mutuo respeto señalada en la propia ley, ya que el hostigamiento dirigido a otras trabajadoras (o trabajadores) incide también en forma negativa en quienes lo presencian, que pueden sentirse razonablemente expuestas a ser objeto del mismo trato, o simplemente afectadas por la discriminación que este implica. Por otro lado, si bien se resguarda la posibilidad de que las personas acosadas formulen sus denuncias no al interior de la empresa, sino directamente ante las autoridades laborales -en especial importante cuando quien ejerce el acoso es el empleador-, el procedimiento igualmente está basado en el propio control, regulación y decisiones que adopte el empleador en cada caso.

Si bien, por un lado se establece la obligatoriedad de la adopción de medidas de resguardo por parte del empleador, ya sea al recibir la denuncia o al ser sugeridas estas medidas por la Inspección del Trabajo en los casos en que esta ha recibido la denuncia, por otro lado, la investigación de los hechos denunciados corresponde al propio empleador, a menos que éste decida remitir los antecedentes a la Inspección del Trabajo respectiva. En este sentido, la ley sólo establece como marco para la investigación interna que sea llevada por escrito, se oiga a ambas partes y que éstas fundamenten sus dichos, sin que exista referencia alguna, por ejemplo, a la idoneidad o imparcialidad de quien realiza la investigación. En este punto es preciso tener presente que no 
se trata de un procedimiento de investigación administrativo, sino de uno directamente delegado en particulares, implicando un riesgo para las garantías de debido proceso de las personas afectadas.

Terminada la investigación interna, sus conclusiones deben ser enviadas a la Inspección del Trabajo, para que esta formule sus observaciones. Estas observaciones o las conclusiones de la investigación llevada a cabo por la Inspección deben ponerse en conocimiento del empleador, denunciante y denunciado, correspondiendo el empleador-de acuerdo al mérito del informe- disponer y aplicar las medidas o sanciones que correspondan.

Las sanciones que pueden imponerse son, en primer término, aquellas reguladas en el reglamento interno, esto es, no superiores a amonestación verbal o escrita, o multa de hasta el $25 \%$ de la remuneración diaria del trabajador denunciado ${ }^{20}$. A ellas se suma la sanción contemplada en el artículo $160 \mathrm{~N}^{\circ} 1$ letra b) del Código del Trabajo, esto es, la facultad del empleador de invocar como causal de caducidad del contrato la realización de conductas de acoso sexual. Es relevante aquí hacer presente que dependerá de la voluntad del empleador la imposición de estas sanciones, e incluso, en casos de acoso sexual grave y reiterado, no es posible sostener que el empleador esté obligado a aplicar la causal de caducidad del contrato. En los casos en que un despido sobre la base de esta causal sea declarado injusto, indebido o improcedente por los Tribunales, el empleador no estará obligado a pagar el recargo de la indemnización correspondiente, si ha cumplido con las obligaciones relativas a las normas del reglamento interno, así como las referidas a la investigación y sanción del acoso sexual ${ }^{21}$.

La situación, por cierto, es más compleja en los casos en que es el propio empleador quien comete el acoso. La nueva ley no logra avanzar mucho más que una incipiente jurisprudencia previa en relación al llamado "despido indirecto" y, en consecuencia, la persona afectada sólo tiene como alternativa al acoso poner término a la relación laboral. En estos casos, la víctima tiene derecho a indemnización aumentada hasta en un $80 \%$. Sin embargo, esta responsabilidad existirá sólo cuando el empleador no hubiera observado el procedimiento de investigación y sanción del acoso sexual establecido en el Título IV del Libro II del Código del Trabajo, procedimiento que, como sabemos, puede ser realizado como una mera formalidad -para evitar esta sanción- sin necesariamente respetar las mínimas garantías de las personas afectadas.

En una realidad de alto desempleo femenino, y considerando que la mayor parte de las mujeres acosadas lo son al comenzar una nueva actividad laboral, la posibilidad de utilizar la figura del "despido indirecto" en la práctica no es una alternativa para las trabajadoras. Equivale a perder un empleo que probablemente fue difícil encontrar, y a obtener una indemnización que, además de tardía, a lo mejor será muy escasa. A lo anterior se suma, desde una perspectiva más general, el hecho de que esta forma de violencia continúa excluyendo a las mujeres de los espacios de trabajo, vulnerando sus derechos fundamentales en esta esfera.
${ }^{20}$ Artículo $154 \mathrm{~N}^{\circ} 10 \mathrm{del}$ Código del Trabajo.

21 Artículo 168 inciso 3으, en relación al artículo 153 inciso $2^{\circ}$ y Título IV del Libro II del Código del Trabajo. 
La exclusión de las Fuerzas Armadas de la normativa sobre acoso sexual, la cuestión relativa a si la sanción penal (originalmente considerada como falta) podría haber tenido un efecto disuasivo sobre estas conductas, así como otros temas, permanecerán en discusión y probablemente serán abordados en las modificaciones legales que puedan existir sobre esta materia a futuro. La efectiva consideración a las obligaciones establecidas en la Convención de Belém do Pará, especialmente en relación a los procedimientos y sanciones, así como en cuanto al acceso de las mujeres a resarcimiento, reparación u otros medios de compensación justos y eficaces, deben dirigir el espíritu tanto de la jurisprudencia judicial y administrativa que comience a dar lugar esta nueva legislación, como de las próximas propuestas legales sobre el tema.

El seguimiento a la aplicación de esta nueva legislación permitirá dilucidar si, efectivamente, se transforma en una herramienta encaminada a erradicar las prácticas de acoso sexual en el lugar de trabajo o, atendida la debilidad de sus disposiciones, sólo se constituirá en un procedimiento generador de expectativas y frustraciones en quienes son víctimas, terminando éstas en la misma disyuntiva previa a la ley: soportar o renunciar. 